\title{
Frontoparietal Cortical Thinning in Respiratory-Type Panic Disorder: A Preliminary Report
}

\author{
Ho-Kyoung Yoon ${ }^{1}$, June Kang ${ }^{2}$, Do-Young Kwon ${ }^{3 凶}$, and Byung-Joo Ham ${ }^{4}$ \\ ${ }^{1}$ Department of Psychiatry, Korea University Ansan Hospital, Ansan, Republic of Korea \\ ${ }^{2}$ Department of Biomedical Science, College of Medicine, Korea University, Seoul, Republic of Korea \\ ${ }^{3}$ Department of Neurology, Korea University Ansan Hospital, Ansan, Republic of Korea \\ ${ }^{4}$ Department of Psychiatry, Korea University Anam Hospital, Seoul, Republic of Korea
}

Objective Many evidences raise the possibility that the panic disorder (PD) patients with respiratory subtype (RS) may have characteristic structural abnormalities. We aimed to explore the structural differences between PD patients with and without the respiratory symptoms.

Methods Patients with PD were recruited from the Department of Psychiatry at Korea University Anam Hospital. Respiratory subtype (RS) was diagnosed when at least 4 out of 5 of the following respiratory symptoms were present during the panic attack: fear of dying, chest pain/discomfort, shortness of breath, paresthesias, and a choking sensation. We acquired high-resolution MRI scans and used FreeSurfer to obtain a measure of cortical thickness for each patient.

Results Cluster based analysis revealed significantly decreased cortical thickness in the left hemisphere in the caudal-middle-frontal, superior frontal, and posterior parietal areas in the RS group. No significant difference was observed in any of the limbic areas.

Conclusion Respiratory symptoms of panic disorder were associated with a reduction in cortical thickness in the left frontal and parietal areas. This finding leads to the assumption that the frontoparietal network is the crucial component in a larger cortical network underlying the perception of dyspnea in RS.

Psychiatry Investig 2016;13(1):146-151

Key Words Cortical thickness, FreeSurfer, Panic disorder, Respiratory type.

\section{INTRODUCTION}

Panic disorder (PD) is diagnosed when an individual experiences recurrent and spontaneous panic attacks (PAs), which characteristically begin with a sudden unexpected rise of terror associated with many autonomic symptoms, particularly cardiorespiratory symptoms. ${ }^{1}$ Despite ongoing research, the pathophysiology of this disorder is yet not well understood. According to the Diagnostic and Statistical Manual of Mental Disorders (DSM-IV), PD is a unitary diagnostic category. ${ }^{2}$ Nevertheless, within this category there are diverse clinical presentations, leading to concerns that $\mathrm{PD}$ is not a singular di-

Received: March 19, 2015 Revised: June 2, 2015

Accepted: June 27, 2015 Available online: October 19, 2015

$\triangle$ Correspondence: Do-Young Kwon, MD, PhD

Department of Neurology, Korea University Ansan Hospital, 123 Jeokgeum-ro, Danwon-gu, Ansan 15355, Republic of Korea

Tel: +82-31-412-5150, Fax: +82-31-412-5154

E-mail: kwondoya@korea.ac.kr

(a) This is an Open Access article distributed under the terms of the Creative Commons Attribution Non-Commercial License (http://creativecommons.org/licenses/bync/3.0) which permits unrestricted non-commercial use, distribution, and reproduction in any medium, provided the original work is properly cited. agnosis. ${ }^{3}$ As a response to these concerns, alternative classification schemes have been devised. Klein ${ }^{4}$ proposed two PD subtypes: a respiratory subtype (RS) with prominent respiratory symptoms, probably linked to a maladaptive false suffocation alarm system, and the autonomic subtype, with prominent peripheral autonomic symptoms such as palpitations. Other authors have proposed that the subtypes should be defined by the most prominent symptoms during a typical PA, such as respiratory, cardiovascular, and gastrointestinal subtypes. ${ }^{5}$

Briggs et al. ${ }^{6}$ studied the psychopathology of 1,034 PD patients and found a high prevalence of respiratory symptoms. In the first part of that study, the PD symptoms of all patients were analyzed with a principal component analysis, a mathematical procedure similar to factor analysis that transforms a number of possibly correlated variables into a smaller number of uncorrelated variables called principal components. This analysis separated two principal components: the first included eight PD symptoms, whereas the second included a fear of dying, chest pain/discomfort, shortness of breath, paresthesias, and a choking sensation. The first principal com- 
ponent included most of the DSM-IV PD symptoms and was correlated to the severity of PD. The second principal component defined a distinct PD subtype, and the absence of these symptoms defined another subtype. When at least four of the five symptoms from the second principal component were present in a given patient, he or she was considered to fall within the RS group. The RS group suffered more spontaneous PAs and seemed to respond better to antidepressants, whereas patients with a non-respiratory subtype (NRS) had more situational PAs and seemed to respond better to benzodiazepines.

In a study by Colasanti and colleagues, mixtures of $\mathrm{CO}_{2}$ were administered to 64 control subjects, and higher concentrations of $\mathrm{CO}_{2}$ produced more anxiety and $\mathrm{PD}$ symptoms. ${ }^{7}$ The factor analysis extracted three components: the respiratory component was mainly characterized by shortness of breath and choking, but also included dizziness, sweating, and palpitations; the neurovegetative component included trembling, chest pain, chills, nausea, and paresthesias; and cognitive factors included fear of dying, fear of losing control, and derealization. The respiratory component symptoms were correlated with $\mathrm{CO}_{2}$-evoked subjective disturbance, as represented by scores on the electronic Visual Analogue Scale of Anxiety.

Accordingly, Nardi et al. ${ }^{8}$ observed that patients responding to a voluntary hyperventilation challenge in the laboratory also reported more respiratory symptoms during attacks in their daily life, and Biber and Alkin ${ }^{9}$ found that a respiratory subgroup of patients was more sensitive to the $\mathrm{CO}_{2}$ challenge. Moreover, respiratory subgroups have been shown to have later panic onset, longer illness duration, higher cigarette consumption, and increased agoraphobia. ${ }^{89}$ Because a higher prevalence of asthma occurs in panic patients, this constellation of respiratory symptoms may be linked to this comorbidity.

$\mathrm{RS}$ patients often have a familial history of $\mathrm{PD},{ }^{10}$ indicating a potential role of genetic factors. Two studies on the genetics of PD suggested that RS may be an important biological marker, although these studies did not address this question directly. ${ }^{11}$ This neurobiological evidence in patients with $\mathrm{RS}$ raises the possibility of characteristic structural abnormalities. However, previous studies have not resolved whether RS and NRS are distinct PD subtypes with a well-defined phenotype. Therefore, it is important to determine whether there are any clinical or other individual differences that may be predictive of a person's panic subtype.

The aim of this study was to identify cortical structural differences between patients with RS and NRS subtypes of PD. Requiring no conscious thought, respiration happens spontaneously following the rhythmic nerve impulses generated and regulated by a group of pacemaker cells in the medulla oblongata and pons. However, breathing can also change in re- sponse to changes in emotions. Final respiratory output is influenced by a complex interaction between the brainstem and higher centers, including the limbic system and cortical structures. Gorman et al. ${ }^{12}$ proposed a significant role of a 'fear network' that is centered in the limbic system such as amygdala, hippocampus and medial frontal cortex. Excessive activity in this network leads to autonomic and neuroendocrine activation through the projections to the brainstem and hypothalamus from the amygdala resulting in the typical PD symptoms. Although limbic system has been play a significant role in the common panic disorder pathophysiology, it is speculated that another area would be involved in distinctive respiratory symptom. Especially, neocortex area could be best candidate, because it receives afferent signals from respiratory system and transfer those signal to other areas. Therefore, we hypothesized that decreased cortical thickness of neocortex structures involved in respiratory perception and control would distinguish patients with RS from those with NRS.

\section{METHODS}

\section{Subjects and procedures}

The study population consisted of twenty one adult patients with PD ages 18 to 65 who were recruited from the Department of Psychiatry at the Korea University Anam Hospital. All participants were right-handed as assessed by the Edinburgh Handedness Test. ${ }^{13}$

Patients who met the criteria for diagnosis of PD as defined by the Diagnostic and Statistical Manual for Mental Disorders, Fourth Edition (DSM-IV), ${ }^{2}$ were recruited for participation. For diagnosing psychiatric disorders, the Korean version of the Mini-International Neuropsychiatric Interview $(\mathrm{MINI})^{14}$ was administered by board certified psychiatric professionals. The patients were diagnosed with RS or NRS according to the criteria described by Briggs et al. ${ }^{6}$ RS was diagnosed when at least 4 out of the following 5 respiratory symptoms were present during the panic attack: fear of dying, chest pain/discomfort, shortness of breath, paresthesias, and a choking sensation.

Exclusion criteria for patients with PD were as follows: 1) any past or current comorbid psychiatric disorders such as mood disorders, psychotic disorders, anxiety disorders, substance-related disorders, and personality disorders, 2) intelligence quotient under 80, 3) clinically significant general and/ or neurological disease which may influence brain structure on imaging, 4) pregnancy or breastfeeding, and 5) any contraindication to undergoing magnetic resonance imaging (MRI) such as the presence of a pacemaker.

After complete explanation of the study to all participants, written informed consent was obtained. The study procedures 
were approved by the Institutional Review Board of the Korea University Anam Hospital. This study was carried out in accordance with the Declaration of Helsinki as revised in 1989.

\section{Assessment for psychopathology}

In order to assess the severity of panic symptoms, the panic disorder severity scale (PDSS $)^{15}$ were used. For the evaluation of the anxiety symptoms, Hamilton anxiety rating scale (HAM-A), ${ }^{16}$ the Spielberger state-trait anxiety inventory (STAI ${ }^{17}$ and anxiety sensitivity index, revised version (ASI-R), ${ }^{18}$ were used.

\section{MRI}

Structural brain images were obtained from three-dimensional MRI scans using a 3.0 Tesla Magnetom Trio Tim System (Siemens Medical Solutions, Inc., Iselin, NJ, USA) at the Korea University Brain Imaging Center. The images were taken with a high resolution T1-weighted Magnetization Prepared Rapid Acquisition Gradient Echo (MP-RAGE) sequence (1900 ms repetition time, $2.6 \mathrm{~ms}$ echo time, $220 \mathrm{~mm}$ field of view, $256 \times 256$ matrix size, 176 coronal slices without gap, $1 \times 1 \times 1 \mathrm{~mm}^{3}$ voxels, $16^{\circ}$ flip angle, number of excitations $=1$ ).

\section{MRI processing for cortical thickness}

Cortical thickness, defined as the shortest distance between the gray/white matter boundary and the spiral surface at each point across the cortical mantle, was automatically estimated by FreeSurfer (software version 5.0, http://surfer.nmr.mgh. harvard.edu). ${ }^{19}$ The technical details of these procedures have been described elsewhere. ${ }^{20}$ Briefly, the process consists of correction for motion artifact, averaging over multiple T1 images, removal of non-brain tissue, Talairach transformation, white matter and subcortical gray matter segmentation, tessellation of the gray matter-white matter boundary, automatic correction for topological defects, intensity normalization, surface deformation, and parcellation of the cerebral cortex into anatomical regions. Total intracranial volume (TIV) was also automatically calculated using FreeSurfer software. ${ }^{21}$ Through careful inspection of all raw images at segmented and inflated stages, we confirmed that none of them exhibited substantial defects. For further analysis, cortical maps were smoothed using a Gaussian kernel with a full width at a half maximum of $10 \mathrm{~mm}$.

\section{Statistical analysis}

Demographic and clinical variables were compared between groups using Mann-Whitney test or $\chi^{2}$ tests for dichotomous variables. All statistical analyses of demographic and clinical data were conducted using SPSS 18.0 (Chicago, IL, USA). The thickness measurement of each vertex was mapped onto a common spherical coordinate system using a spherical transformation. Maps were smoothed using a $10 \mathrm{~mm}$ Gaussian kernel. A general linear model controlled for the effects of sex, age, and ICV in the estimation of differences in cortical thickness between the groups with and without respiratory symptoms at each vertex. The right and left hemispheres were tested separately. Monte Carlo simulations were performed in order to identify contiguous clusters of significant vertex-wise group differences [family-wise error (FWE) corrected, $\mathrm{p}<0.05$ ].

\section{RESULTS}

\section{Demographic and clinical variables}

A total of 11 RS and 10 NRS patients were recruited to participate in this study. The demographic and clinical variables for each group are presented in Table 1. There were no significant differences in age or gender between groups. Scales for panic and general anxiety showed no statistical differences between groups.

\section{FreeSurfer}

Cortical thickness was decreased in the RS group in a circumscribed cluster ( $p<0.05$, corrected) located in the left caudal middle frontal cortex, which is part of the premotor cor-

Table 1. Sociodemographic and clinical characteristics

\begin{tabular}{lcccc}
\hline & RS $(\mathrm{N}=11)$ & NRS $(\mathrm{N}=10)$ & Statistics & p-value \\
\hline Age $(\mathrm{y})^{*}$ & $37.27(9.36)$ & $43.70(12.283)$ & 38.50 & 0.251 \\
Gender, female $^{\dagger}$ & $7(58.3)$ & $5(50)$ & 0.528 & 0.398 \\
PDSS* $^{*}$ & $9.40(4.62)$ & $8.60(5.06)$ & 43.00 & 0.631 \\
HAM-A $^{*}$ & $18.73(11.57)$ & $12.60(8.15)$ & 35.00 & 0.173 \\
STAIX-S* $^{*}$ & $44.73(4.34)$ & $43.90(9.39)$ & 44.00 & 0.468 \\
STAIX-T* $^{*}$ & $45.45(8.51)$ & $40.10(10.47)$ & 31.50 & 0.099 \\
\hline
\end{tabular}

Data are presented as means (SD) or numbers (\%). ${ }^{*}$ Mann-Whitney $U$ test. Statistics, $U$, ${ }^{\dagger}$ chi-square $\left(\chi^{2}\right)$ test was used. RS: respiratory subtype, NRS: non-respiratory subtype, PDSS: panic disorder severity scale, HAM-A: Hamilton anxiety rating scale, STAIX-S: state trait anxiety inventory-state, STAIX-T: state trait anxiety inventory-trait 
tex, in comparison to the NRS group (Figure 1). The cluster was $688.43 \mathrm{~mm}^{2}$ in size and MNI coordinates at the maximum were $-33.4,-0.3,47.4(\mathrm{x}, \mathrm{y}, \mathrm{z})$ (Table 2).

Significantly thinner cortex in the RS group was also found in a confined cluster (Figure 1), located within the left superior frontal cortex (cluster size: $1329.51 \mathrm{~mm}^{2}$, MNI coordinates at the maximum: $-6.9,48.7,40.5$ ) and the left posterior parietal cortex (cluster size: $791.45 \mathrm{~mm}^{2}$, MNI coordinates at the maximum: $-19.9,-62.1,39.8$ ) (Table 2).

\section{DISCUSSION}

Our major findings were that cortical thickness in the middle frontal, superior frontal, and posterior parietal areas of the left hemisphere were decreased in the RS group compared to the NRS group. It was suggested that dysfunction of these cortical areas might represent a crucial pathophysiological mechanism of RS. No significant difference was observed in any of the limbic areas. Limbic areas are supposed to be the common panic disorder pathophysiology. To the best of our knowledge, this study is first structural MRI studies examining possible differences in gray matter structure in RS.

Despite the use of different intervention techniques, common activations in frontal areas have been demonstrated in the several dyspnea imaging studies. In the previous stud-
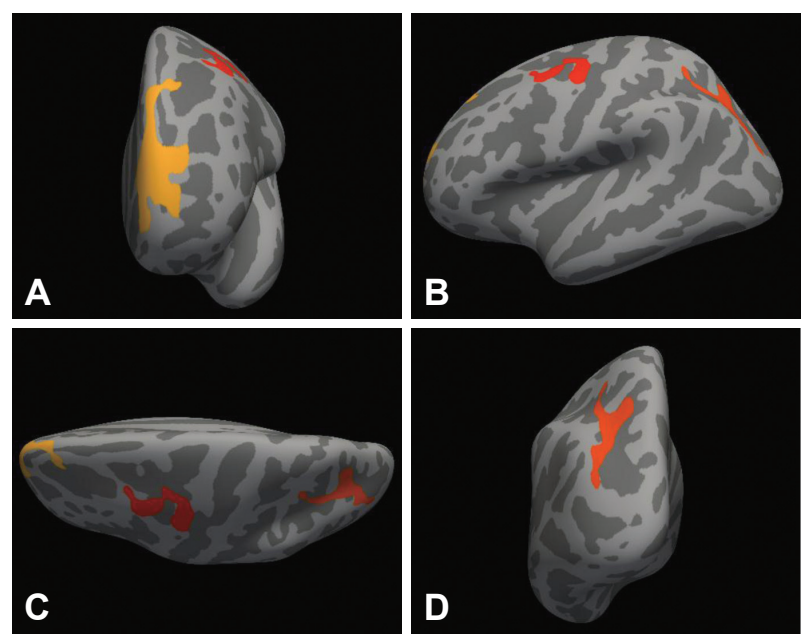

Figure 1. Location of decreased cortical thickness in patients with respiratory subtype panic disorder compared to the non-respiratory subtype. A: Rosral view. B: Lateral view. C: Dorsal view. D: Caudal view. $p<0.05$ (FWE-corrected). FEW: family-wise error. ies, ${ }^{22,23}$ breathlessness was induced in nine healthy volunteers by the inspiration of increased $\mathrm{CO}_{2}(8 \%)$ using either a facemask or a mouthpiece. In the study of Evans et al., ${ }^{24}$ they induced breathlessness in six healthy mechanically ventilated participants by restraining the tidal volume below spontaneous levels in combination with constantly elevating arterial $\mathrm{PCO}_{2}$ levels by manipulating inspired $\mathrm{PcO}_{2}$, and compared dyspneic conditions with episodes of higher tidal volume combined with normal arterial $\mathrm{PcO}_{2}$, which relieved breathlessness. Hypercapnia in particular activates the middle frontal gyrus ${ }^{22}$ and co-activates the prefrontal cortex..$^{25}$ Restricted tidal volume below resting levels (Low VT) activates the superior frontal gyrus. ${ }^{24}$ This activation may be the direct result of stimulating common lung and chest wall mechanoreceptors activated by changes in thoracic volume. Otherwise, the frontal lobe may play an important role in assigning emotional meaning to the respiratory experience because it is implicated in the retention of memories associated with emotions derived from limbic input. ${ }^{26}$

Inspiratory and expiratory loads as well as low VT activate discrete regions of the posterior parietal lobule. ${ }^{23}$ The posterior parietal cortex receives and integrates information from the somatosensory area, and sends this information on to motor areas. This area is itself closely interconnected with the prefrontal areas, and together these two regions represent the highest level of integration in the motor control hierarchy. It is here that the decisions are made about what actions will be taken. Our study identified prominent thinning in the posterior parietal cortex as well as in the prefrontal and premotor cortices, primary components of the frontoparietal network (FPN). Different brain regions show a high temporal correlation in their activity which has led to the hypothesis that the brain forms intrinsic connectivity networks (ICN). ${ }^{27,28}$ The term ICN describes a set of brain regions that are functionally interconnected, even when the brain is not probed by external stimulation. Thus, the activity is not a reaction to some sort of task but intrinsically generated by the brain itself. Multiple functional networks, including default-mode network, have been identified with high reliability. ${ }^{29-31}$ Of these ICNs, the FPN has been associated with executive functions, such as attention, memory, and inhibitory control. ${ }^{32,33}$ Moreover, this network has been associated with global dimensions of emotional functioning as well as with anxiety traits. ${ }^{34-36}$

Table 2. Cluster-based analysis

\begin{tabular}{|c|c|c|c|c|c|c|c|}
\hline \multirow{2}{*}{ Cortical area } & \multirow{2}{*}{\multicolumn{3}{|c|}{ Talairach coordinates }} & \multirow{2}{*}{ BA } & \multicolumn{2}{|c|}{ Mean (SD) in $\mathrm{mm}$} & \multirow{2}{*}{ p-value } \\
\hline & & & & & Symptom $+(\mathrm{N}=11)$ & Symptom- $(\mathrm{N}=10)$ & \\
\hline Left caudal middle frontal cortex & -33.0 & 1.9 & 43.6 & 6 & $2.58(0.23)$ & $2.66(0.17)$ & 0.039 \\
\hline Left superior frontal & -6.8 & 49.0 & 34.9 & 9 & $2.80(0.21)$ & $2.83(0.12)$ & 0.000 \\
\hline Left posterior parietal & -19.7 & -58.3 & 39.5 & 7 & $2.31(0.19)$ & $2.35(0.18)$ & 0.016 \\
\hline
\end{tabular}


Our results suggest that respiratory discomfort in RS patients may arise from disturbances in modulation of the perceived intensity of sensations by factors other than the primary mechanism, which may include emotional processing. Although the amygdala receives direct sensory inputs from brainstem structures and the sensory thalamus that enable a rapid response to potentially threatening stimuli, it also receives afferents from cortical regions involved in the processing and evaluation of sensory information. ${ }^{37}$ The posterior parietal cortex has historically been considered a typical association cortex, important for spatial attention and multisensory integration in the generation of a unitary map whose output is relayed to the frontal lobe to guide behavior. ${ }^{38}$ Frontoparietal area has a unique set of reciprocal connections with limbic and sensory areas. ${ }^{39,40}$ In other words, besides common limbic fear network, FPN would play an important role in pathomechanism of RS subtype PD. This can be new causal model of respiratory phenotype PD.

There are several limitations of the current study that should be noted. First, the sample size is small, primarily due to our stringent inclusion and exclusion criteria. Patients with PD frequently have comorbid psychiatric disorders, such as major depressive disorder, ${ }^{41}$ alcohol abuse ${ }^{42}$ and bipolar disorder. ${ }^{43}$ Additionally, these psychiatric conditions are known to be associated with structural abnormalities in the brain. We believe that the absence of a comorbid anxiety disorder might contribute to the validity of this study, since most of the anxiety spectrum disorders are associated with disturbed fear circuitry composed of the amygdala, medial prefrontal cortex, insula, and hippocampal formation. ${ }^{44}$ Thus, the small sample size included in this study allowed us to minimize any confounding factors which might have influenced our structural data. Another weakness of this study is the cross-sectional design based on retrospective reports. Additionally, treatment data were not collected and periods of PD exacerbation or remission were not assessed. Lack of normal control values from healthy volunteers is a limitation of this study. This also limit interpretation of this study. Lastly, in using Briggs' classification, patients with many symptoms have an increased chance of being classified as respiratory subtype. On the other hand, if a patient has strong feelings of choking, shortness of breath, and chest discomfort but does not experience paresthesias and fear of dying, she or he will not be diagnosed with the respiratory subtype. Although consistent findings are reported in patients with the respiratory subtype such as a positive family history and high sensitivity to $\mathrm{CO}_{2}$ challenge tests, ${ }^{10}$ many studies report conflicting findings regarding the age of onset and comorbidities. ${ }^{45}$ These inconsistencies may be due to the limitations of Briggs' classification. Other dimensional taxonomy may also be helpful in identifying the core symp- toms and in capturing the subtle psychopathology of anxiety disorders. ${ }^{46}$

In conclusion, we demonstrated decreased cortical thickness in a confined area in the left superior and caudal middle frontal cortex and posterior parietal cortex in RS patients. These areas were related to respiratory perception and emotion regulation, possibly reflecting emotional dysregulation in RS. This finding suggested that the frontotemporal network is a crucial component within a larger brain network underlying the perception of dyspnea. Although the sample size of this study is small, we believe that our results help to elucidate the etiological characteristics of RS, thereby improving therapeutic approaches in this population. Further studies with larger sample sizes and additional subtypes of PD are necessary to confirm and expand on our findings.

\section{Acknowledgments}

This work was supported by a grant from Korea University (K1326261); Lee Byung Yoon Grant.

\section{REFERENCES}

1. Roberson-Nay R, Kendler KS. Panic disorder and its subtypes: a comprehensive analysis of panic symptom heterogeneity using epidemiological and treatment seeking samples. Psychol Med 2011;41:2411-2421.

2. American Psychiatric Association. Diagnostic and Statistical Manual of Mental Disorders, Fourth Edition. Washington DC: American Psychiatric Association; 1994.

3. Beck JG, Shipherd JC, Ohtake P. Do panic symptom profiles influence response to a hypoxic challenge in patients with panic disorder? A preliminary report. Psychosom Med 2000;62:678-683.

4. Klein DF. False suffocation alarms, spontaneous panics, and related conditions. An integrative hypothesis. Arch Gen Psychiatry 1993;50: 306-317.

5. Aronson TA, Logue CM. Phenomenology of panic attacks: a descriptive study of panic disorder patients' self-reports. J Clin Psychiatry 1988; 49:8-13.

6. Briggs AC, Stretch DD, Brandon S. Subtyping of panic disorder by symptom profile. Br J Psychiatry 1993;163:201-209.

7. Colasanti A, Salamon E, Schruers K, van Diest R, van Duinen M, Griez EJ. Carbon dioxide-induced emotion and respiratory symptoms in healthy volunteers. Neuropsychopharmacology 2008;33:3103-3110.

8. Nardi AE, Lopes FL, Valenca AM, Nascimento I, Mezzasalma MA, Zin WA. Psychopathological description of hyperventilation-induced panic attacks: a comparison with spontaneous panic attacks. Psychopathology 2004;37:29-35.

9. Biber B, Alkin T. Panic disorder subtypes: differential responses to CO2 challenge. Am J Psychiatry 1999;156:739-744.

10. Nardi AE, Valenca AM, Nascimento I, Lopes FL, Mezzasalma MA, Freire RC, et al. A three-year follow-up study of patients with the respiratory subtype of panic disorder after treatment with clonazepam. Psychiatry Res 2005;137:61-70.

11. Smoller JW, Tsuang MT. Panic and phobic anxiety: defining phenotypes for genetic studies. Am J Psychiatry 1998;155:1152-1162.

12. Gorman JM, Kent JM, Sullivan GM, Coplan JD. Neuroanatomical hypothesis of panic disorder, revised. Am J Psychiatry 2000;157:493-505.

13. Oldfield RC. The assessment and analysis of handedness: the Edinburgh inventory. Neuropsychologia 1971;9:97-113.

14. Sheehan DV, Lecrubier Y, Sheehan KH, Amorim P, Janavs J, Weiller E, et al. The Mini-International Neuropsychiatric Interview (M.I.N.I.): the 
development and validation of a structured diagnostic psychiatric interview for DSM-IV and ICD-10. J Clin Psychiatry 1998;59(Suppl 20): 22-33.

15. Shear MK, Rucci P, Williams J, Frank E, Grochocinski V, Vander Bilt J, et al. Reliability and validity of the Panic Disorder Severity Scale: replication and extension. J Psychiatr Res 2001;35:293-296.

16. Maier W, Buller R, Philipp M, Heuser I. The Hamilton Anxiety Scale: reliability, validity and sensitivity to change in anxiety and depressive disorders. J Affect Disord 1988;14:61-68.

17. Spielberger CD, Gorsuch RL, Lushene RE. Manual for the State-Trait Anxiety Inventory. Palo Alto, CA: Consulting Psychologist Press; 1970.

18. Lim YJ, Yu BH, Kim JH. Korean Anxiety Sensitivity Index-Revised: its factor structure, reliability, and validity in clinical and nonclinical samples. Depress Anxiety 2007;24:331-341.

19. Nakamura K, Fox R, Fisher E. CLADA: cortical longitudinal atrophy detection algorithm. Neuroimage 2011;54:278-289.

20. Fischl B, Dale AM. Measuring the thickness of the human cerebral cortex from magnetic resonance images. Proc Natl Acad Sci U S A 2000; 97:11050-11055.

21. Jovicich J, Czanner S, Han X, Salat D, van der Kouwe A, Quinn B, et al. MRI-derived measurements of human subcortical, ventricular and intracranial brain volumes: reliability effects of scan sessions, acquisition sequences, data analyses, scanner upgrade, scanner vendors and field strengths. Neuroimage 2009;46:177-192.

22. Liotti M, Brannan S, Egan G, Shade R, Madden L, Abplanalp B, et al. Brain responses associated with consciousness of breathlessness (air hunger). Proc Natl Acad Sci U S A 2001;98:2035-2040.

23. Brannan S, Liotti M, Egan G, Shade R, Madden L, Robillard R, et al. Neuroimaging of cerebral activations and deactivations associated with hypercapnia and hunger for air. Proc Natl Acad Sci U S A 2001; 98:2029-2034.

24. Evans KC, Banzett RB, Adams L, McKay L, Frackowiak RS, Corfield DR. BOLD fMRI identifies limbic, paralimbic, and cerebellar activation during air hunger. J Neurophysiol 2002;88:1500-1511.

25. Corfield DR, Fink GR, Ramsay SC, Murphy K, Harty HR, Watson JD, et al. Activation of limbic structures during $\mathrm{CO} 2$-stimulated breathing in awake man. Adv Exp Med Biol 1995;393:331-334.

26. Miller EK, Cohen JD. An integrative theory of prefrontal cortex function. Annu Rev Neurosci 2001;24:167-202.

27. Friston KJ, Frith CD, Liddle PF, Frackowiak RS. Functional connectivity: the principal-component analysis of large (PET) data sets. J Cereb Blood Flow Metab 1993;13:5-14.

28. Biswal B, Yetkin FZ, Haughton VM, Hyde JS. Functional connectivity in the motor cortex of resting human brain using echo-planar MRI. Magn Reson Med 1995;34:537-541.

29. Shehzad Z, Kelly AM, Reiss PT, Gee DG, Gotimer K, Uddin LQ, et al. The resting brain: unconstrained yet reliable. Cereb Cortex 2009;19: 2209-2229.

30. Damoiseaux JS, Rombouts SA, Barkhof F, Scheltens P, Stam CJ, Smith $\mathrm{SM}$, et al. Consistent resting-state networks across healthy subjects.
Proc Natl Acad Sci U S A 2006;103:13848-13853.

31. De Luca M, Beckmann CF, De Stefano N, Matthews PM, Smith SM. fMRI resting state networks define distinct modes of long-distance interactions in the human brain. Neuroimage 2006;29:1359-1367.

32. Posner MI, Dehaene S. Attentional networks. Trends Neurosci 1994; 17:75-79.

33. Mesulam MM. Large-scale neurocognitive networks and distributed processing for attention, language, and memory. Ann Neurol 1990;28: 597-613.

34. Chabernaud C, Mennes M, Kelly C, Nooner K, Di Martino A, Castellanos FX, et al. Dimensional brain-behavior relationships in children with attention-deficit/hyperactivity disorder. Biol Psychiatry 2012;71: 434-442.

35. Etkin A, Prater KE, Schatzberg AF, Menon V, Greicius MD. Disrupted amygdalar subregion functional connectivity and evidence of a compensatory network in generalized anxiety disorder. Arch Gen Psychiatry 2009;66:1361-1372.

36. Qiu C, Liao W, Ding J, Feng Y, Zhu C, Nie X, et al. Regional homogeneity changes in social anxiety disorder: a resting-state fMRI study. Psychiatry Res 2011;194:47-53.

37. Mezzasalma MA, Valenca AM, Lopes FL, Nascimento I, Zin WA, Nardi AE. Neuroanatomy of panic disorder. Rev Bras Psiquiatr 2004;26: 202-206.

38. Colby CL, Goldberg ME. Space and attention in parietal cortex. Annu Rev Neurosci 1999;22:319-349.

39. Cavada C, Goldman-Rakic PS. Posterior parietal cortex in rhesus monkey: II. Evidence for segregated corticocortical networks linking sensory and limbic areas with the frontal lobe. J Comp Neurol 1989; 287:422-445.

40. Cavada C, Goldman-Rakic PS. Posterior parietal cortex in rhesus monkey: I. Parcellation of areas based on distinctive limbic and sensory corticocortical connections. J Comp Neurol 1989;287:393-421.

41. Kessler RC, Stang PE, Wittchen HU, Ustun TB, Roy-Burne PP, Walters EE. Lifetime panic-depression comorbidity in the National Comorbidity Survey. Arch Gen Psychiatry 1998;55:801-808.

42. Roberts JM, Malcolm R, Santos AB. Treatment of panic disorder and comorbid substance abuse with divalproex sodium. Am J Psychiatry 1994;151:1521.

43. Kilbane EJ, Gokbayrak NS, Galynker I, Cohen L, Tross S. A review of panic and suicide in bipolar disorder: does comorbidity increase risk? J Affect Disord 2009;115:1-10.

44. Shin LM, Liberzon I. The neurocircuitry of fear, stress, and anxiety disorders. Neuropsychopharmacology 2010;35:169-191.

45. Kircanski K, Craske MG, Epstein AM, Wittchen HU. Subtypes of panic attacks: a critical review of the empirical literature. Depress Anxiety 2009;26:878-887.

46. Grant BF, Hasin DS, Stinson FS, Dawson DA, Goldstein RB, Smith S, et al. The epidemiology of DSM-IV panic disorder and agoraphobia in the United States: results from the National Epidemiologic Survey on Alcohol and Related Conditions. J Clin Psychiatry 2006;67:363-374. 